\title{
$\mathrm{CaAl}$ 类水滑石的磷酸根吸附性能及其影响因素研究
}

\author{
贾云生 ${ }^{a, b}$ 王火焰 ${ }^{*, a}$ 赵雪松 ${ }^{c}$ 刘晓伟 ${ }^{a, b}$ \\ 王一柳 ${ }^{a} \quad$ 范群龙 ${ }^{c}$ 周健民 ${ }^{a}$ \\ ( ${ }^{a}$ 中国科学院南京土壤研究所土壤与农业可持续发展国家重点实验室 南京 210008) \\ $\left({ }^{b}\right.$ 中国科学院大学 北京 100049) \\ (c 江苏隆昌化工有限公司 江苏 南通 226500)
}

\begin{abstract}
摘要 本研究采用共沉淀法成功制备了 $\mathrm{CaAl}$ 类水滑石 $(\mathrm{CaAl}-\mathrm{LDH})$, 并运用扫描电子显微镜(SEM)和热重/差示扫描量 热分析(TG-DSC)技术对 CaAl-LDH 的形貌及热稳定性进行了表征. 利用合成含磷溶液及真实含磷污水, 探究了 CaAl-LDH 对磷酸根的吸附性能及其主要影响因素. 结果表明: 制备的 CaAl-LDH 具备类水滑石的典型六边形层片状结 构以及较好的热稳定性. CaAl-LDH 吸附磷酸根的规律符合 Langmuir 吸附等温方程, 理论最大吸附容量为 $162.3 \mathrm{mg} / \mathrm{g}$. 吸附动力学过程符合假二级动力学方程. 几种竞争阴离子对吸附的干扰作用由强到弱为 $\mathrm{CO}_{3}^{2-}>\mathrm{SO}_{4}^{2-}>\mathrm{NO}_{3}^{-}$. 吸附 真实含磷污水中磷酸根时, $10 \mathrm{~h}$ 左右达最大去除率，可将污水中磷浓度降低到极低水平. 因此，制备的 CaAl-LDH 具有 很好的磷酸根吸附性能, 是一种应用前景广阔的污水磷酸根吸附回收利用材料.
\end{abstract}

关键词 $\mathrm{CaAl}-\mathrm{Cl}$ 类水滑石; 磷酸根; 高效吸附; 吸附动力学; 影响因素

\section{Exploring and Evaluation of CaAl Hydrotalcite-like Adsorbents on Phosphate Recycling}

\author{
Jia, Yunsheng ${ }^{a, b}$ \\ Wang, Huoyan ${ }^{*, a}$ \\ Zhao, Xuesong ${ }^{c}$ \\ Liu, Xiaowei ${ }^{a, b}$ \\ Wang, Yiliu ${ }^{a}$ Fan, Qunlong ${ }^{c}$ Zhou, Jianmin ${ }^{a}$ \\ ( ${ }^{a}$ State Key Laboratory of Soil and Sustainable Agriculture, Institute of Soil Science, Chinese Academy of Sciences, \\ Nanjing 210008) \\ ( ${ }^{b}$ University of the Chinese Academy of Sciences, Beijing 100049) \\ ( ${ }^{c}$ Jiangsu Longchang Chemical Co., Ltd., Nantong 226500)
}

\begin{abstract}
We synthesized CaAl-Cl layered double hydroxide (CaAl-LDH) by co-precipitation and evaluated its performance in phosphate adsorption. Calcium fluoride $(0.25 \mathrm{~mol})$ and sodium meta-aluminate $(0.1 \mathrm{~mol})$ were poured into a beaker that contained $0.2 \mathrm{~mol}$ sodium hydroxide. The suspension was aged at $25{ }^{\circ} \mathrm{C}$ for $4 \mathrm{~h}$ under vigorous stirring. The acquired sludge was filtered and washed using distilled water, dried at $60{ }^{\circ} \mathrm{C}$, and then ground to powder for further study. Phosphate stock solution was prepared using sodium di-hydrogen phosphate, and used for adsorption kinetic and isotherm research. The impact factors (e.g., competitive anions, adsorbent dosage, and adsorption time) of phosphate removal by CaAl-LDH were researched using industrial effluents. Adsorption processes were studied in $50 \mathrm{~mL}$ centrifuge tubes, with shaking by a thermostatic oscillator for planned time intervals. Mixtures were filtered through a $0.22 \mu \mathrm{m}$ membrane before analysis. Samples before and after phosphate adsorption were characterized by scanning electron microscopy to observe morphological characteristics. Thermo-gravimetry/differential scanning calorimetry was used to study the sample thermo-stability. Metal ions (e.g. calcium, aluminum) and phosphate concentration were determined by inductively coupled plasma atomic emission spectrometry. We observed a typical hydrotalcite structure in the CaAl-LDH by scanning electron microscopy, which indicates that the obtained materials are well-defined hexagonal platelet-like particles. The synthesized CaAl-LDH had good thermo-stability based on thermo-gravimetry/differential scanning calorimetry, and pyrolysis showed four mass loss stages. From batch adsorption experiments, we found that the adsorption fitted a Langmuir model $\left(R^{2}=0.997\right)$ very well; the maximum adsorption capacity $\left(q_{\max }\right)$ of $162.3 \mathrm{mg} / \mathrm{g}$ corresponded to the experimental data $(166.9 \mathrm{mg} / \mathrm{g})$, and is significantly higher than previous reports. The adsorption kinetics followed a pseudo-second-order model $\left(R^{2}=0.998\right)$. Additionally, co-existing competitive anions influenced phosphate adsorption in the order of $\mathrm{CO}_{3}^{2-}>\mathrm{SO}_{4}^{2-}>\mathrm{NO}_{3}^{-}$. Adsorbent dosage and contact time could also impact CaAl-LDH phosphate adsorption capacity. CaAl-LDH in this study could be applied in the recovery and reuse of phosphate from industrial wastewater because of its outstanding performance and stability.
\end{abstract}

Keywords CaAl-Cl layered double hydroxide; phosphate; efficient adsorption; adsorption kinetics; impact factors

* E-mail: hywang@issas.ac.cn

Received July 15, 2015; published August 20, 2015.

Project supported by the National Basic Research Program of China (No. 2013CB127401) and the National Natural Science Foundation of China (No. 41271309).

项目受国家 973 项目(No. 2013CB127401)和国家自然科学基金面上项目(No. 41271309)资助. 


\section{1 引言}

磷素是一种不可再生、不可替代的自然资源, 是工 业生产的重要材料, 也是农作物生长必不可少的营养元 素之一 ${ }^{[1]}$. 然而, 随着世界人口数量以及工农业生产规 模的不断增加, 全球的磷素资源正日趋减少 ${ }^{[2]}$. 据预测, 全世界的磷矿储量将在 50 年内耗竭 ${ }^{[3]}$. 另一方面, 工农 业生产过程排放大量的含磷污水到环境水体, 不仅会导 致水体富营养化 ${ }^{[4 ~ 7]}$, 也会对磷素资源造成极大的浪费. 因此, 近几十年来, 污水中磷素 (磷酸根) 的去除和回收 利用一直是研究的热点. 当前, 污水除磷的方法主要包 括: 化学沉淀法 ${ }^{[8]}$ 、膜技术处理法 ${ }^{[9]} 、$ 生物处理法和吸附 法等 ${ }^{[10]}$. 化学沉淀法操作稳定、去除率较高, 但费用高、 易产生二次污泥且无法应用于低浓度污水 ${ }^{[6,11]}$; 膜技术 处理法的膜通量小、寿命短且易堵塞 ${ }^{[9]}$; 生物处理法处 理速度慢、操作复杂, 且不适用于处理高浓度含磷污

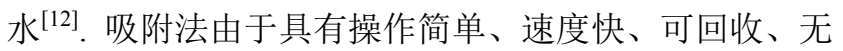
二次污染等优点受到关注 ${ }^{[13,14]}$. 常用的吸附材料可分为 三大类: 天然材料和工矿废渣(如针铁矿、沸石、方解石、 蒙脱石、高炉矿渣、香蕉秸秆等 ${ }^{[, 11,15]}$ )、活性物质及其 改性物(如活性炭、活性氧化铝 ${ }^{[16]}$ 等) 以及人工合成吸附 剂(如类水滑石 ${ }^{[10]}$ 等). 天然材料及活性物质对吸附质缺 乏选择性, 吸附容量低, 再生性差 ${ }^{[17]}$. 目前国内外吸附 研究主要围绕具有高吸附容量、可循环利用以及环境友 好等特点的新型吸附剂的开发 ${ }^{[6,17]}$. 类水滑石在众多领 域(如作为催化剂、基因和药物载体以及阴离子分离等) 都有着广泛应用, 良好的阴离子交换性能令其在吸附和 去除污水阴离子污染物领域具有巨大潜力 ${ }^{[18]}$.

类水滑石 (hydrotalcite-like compounds, HTLc) 又名 层状双金属氢氧化物(layered double hydroxides, LDHs), 组成通式为: $\left[\mathrm{M}_{1-x}^{2+} \mathrm{M}_{x}^{3+}(\mathrm{OH})_{2}\right] \mathrm{A}_{x / n}^{n-} \bullet m \mathrm{H}_{2} \mathrm{O}$, 其中 $\mathrm{M}^{2+}$ 和 $\mathrm{M}^{3+}$ 分别代表二价和三价金属阳离子, $\mathrm{A}^{n-}$ 代表层间 阴离子, $x$ 为 $\mathrm{M}^{3+} /\left(\mathrm{M}^{2+}+\mathrm{M}^{3+}\right)$ 的物质的量比, 一般在 $0.17 \sim 0.33$ 之间 ${ }^{[14,19]}$. LDHs 具有巨大的比表面积, 良好 的阴离子交换性能, 以及特殊的 “记忆效应”, 是去除 水体中阴离子污染物的优良吸附材料[2,6,20,21].

目前关于含 $\mathrm{MgAl}^{[22,23]} 、 \mathrm{ZnAl}^{[24,25]}$ 等金属阳离子的 类水滑石吸附水体中磷酸根的报道很多, 但吸附容量不 高. Goh 等综述了只含一种含氧阴离子的溶液体系中, LDHs 的吸磷容量在 $7.3 \sim 81.6 \mathrm{mg} / \mathrm{g}^{[26]}$. 已有报道, 吸附 于 LDHs 上的磷素可以维持一种绿色海藻一一石花的正 常生长 ${ }^{[27]}$. 说明通过 LDHs 吸附的磷酸根可以实现磷素 的回收再利用. 因此, 磷素的耗竭、污染和回收再利用 等方面, 都迫切要求找到更加高效环保的吸附剂材料. 本研究试图通过共沉淀法 ${ }^{[28]}$ 制备能够高效吸附水体中 磷酸根的 $\mathrm{CaAl}$ 类水滑石, 研究其除磷动力学和吸附等 温线, 分析竞争离子、吸附剂用量以及吸附时间等因素 对吸附过程的影响, 并考察其去除实际化工厂污水中磷 酸根的性能.

\section{2 结果与讨论}

\section{1 表征分析}

通过共沉淀法制备 $\mathrm{CaAl}$ 类水滑石(以下均简称 $\mathrm{CaAl}-\mathrm{LDH}$ ). 图 1 为 $\mathrm{CaAl}-\mathrm{LDH}$ 的扫描电子显微镜(SEM) 图片(放大 10000 倍), 可以看到颗粒表面均匀, 六边形 层片状，且不规则地垛叠聚集在一起，粒径大小在 $0.5 \sim 2 \mu \mathrm{m}$ 左右. 根据 BET 方法测得比表面积为 7.6 $\mathrm{m}^{2} / \mathrm{g}$.

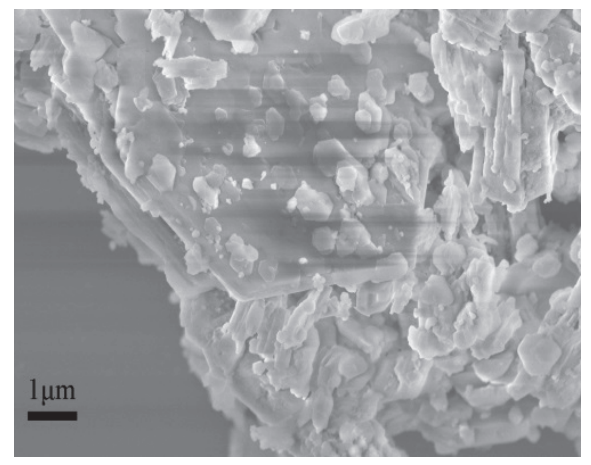

图 $1 \mathrm{CaAl}-\mathrm{LDH}$ 的 SEM 图片

Figure 1 SEM image of CaAl-LDH

CaAl-LDH 的热重/差示扫描量热分析(TG-DSC)结 果如图 2 所示. 其中的 TG 曲线具有四个失重台阶, 并 且 DSC 曲线上存在两个独立的吸热峰, 最大吸热峰值 时的温度分别为 127 和 $404{ }^{\circ} \mathrm{C}$. 说明 CaAl-LDH 的热分 解过程分四个阶段. $134{ }^{\circ} \mathrm{C}$ 之前为第一阶段, 失重 $7.71 \%$, 对应的是层间结晶水的释放; $134 \sim 361{ }^{\circ} \mathrm{C}$ 为第 二阶段, 失重 $9.8 \%$, 此阶段对应的是层间 $\mathrm{Cl}^{-}$的释放; $361 \sim 415{ }^{\circ} \mathrm{C}$ 为第三阶段, 失重 $3.1 \%$, 此阶段可能是合 成过程中混入碳酸根离子, 以 $\mathrm{CO}_{2}$ 的形式脱除; 第四阶 段为 $562{ }^{\circ} \mathrm{C}$ 之后, 对应的是层板羟基的脱除 ${ }^{[29 ~ 31]}$. 由 此可知, 制备的 CaAl-LDH 具有较好的热稳定性.

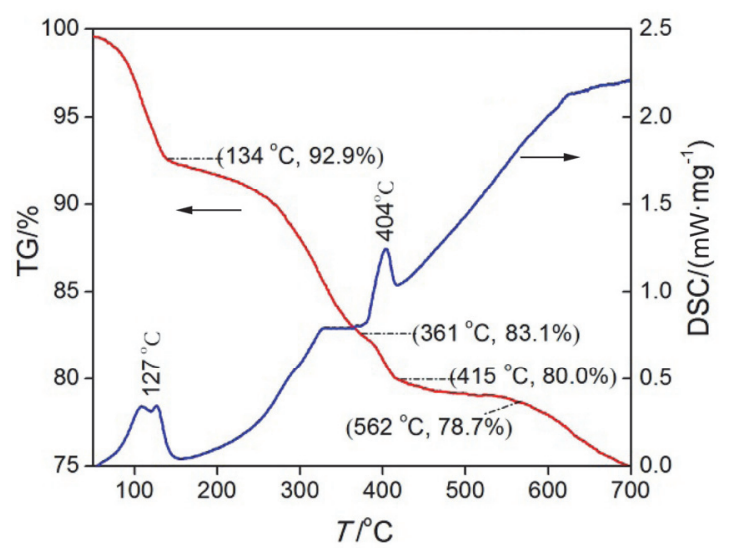

图 $2 \mathrm{CaAl}-\mathrm{LDH}$ 的 TG-DSC 曲线

Figure 2 TG-DSC curve of CaAl-LDH

\section{2 吸附动力学}

向 $500 \mathrm{mg} / \mathrm{L} \mathrm{NaH} \mathrm{PO}_{4}$ 溶液 $(\mathrm{pH}=4.8)$ 中, 加入 2.5 
$\mathrm{g} / \mathrm{L} \mathrm{CaAl-LDH}$, 探究该材料吸附磷酸根的动力学过程. 如图 3 所示, 吸附过程大致分两个阶段 ${ }^{[12]}: 1.5 \mathrm{~h}$ 以内的 快速吸附阶段，此阶段吸附率达到 $52.7 \%$ (本文吸附率均 指质量百分率); $1.5 \mathrm{~h}$ 以后的慢速吸附阶段, 在 $3 \mathrm{~h}$ 左右 时达到吸附平衡, 此时达到磷酸根的最大吸附率 $55.2 \%$.

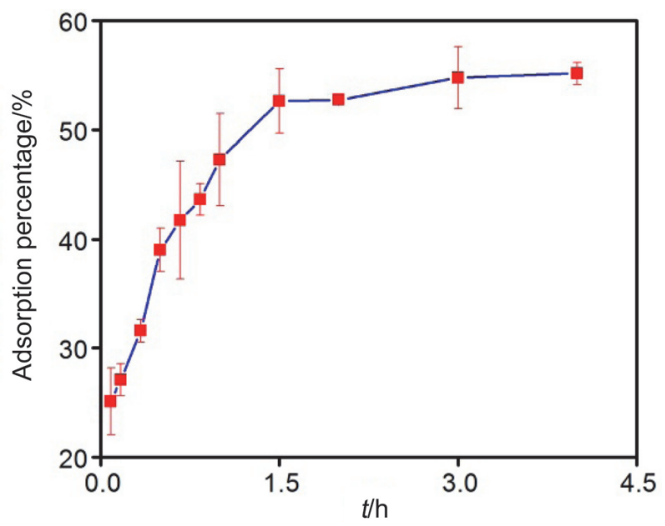

图 3 吸附率随时间变化情况

Figure 3 Adsorption percentage change with time

假一级及假二级反应动力学模型被广泛用于描述 类水滑石吸附阴离子的动力学过程 ${ }^{[28]}$, 分别表示吸附 过程的速率控制步骤为质量传递过程和化学反应过 程 ${ }^{[32]}$. 运用假一级和假二级模型方程对 CaAl-LDH 吸附 磷酸根的动力学实验数据进行拟合, 方程线性表达式如 下:

假一级反应动力学方程 ${ }^{[33]}$ :

$$
\ln \left(q_{\mathrm{e}}-q_{t}\right)-\ln \left(q_{\mathrm{e}}\right)=-k_{1} * t / 2.303
$$

假二级反应动力学方程 ${ }^{[33]}$.

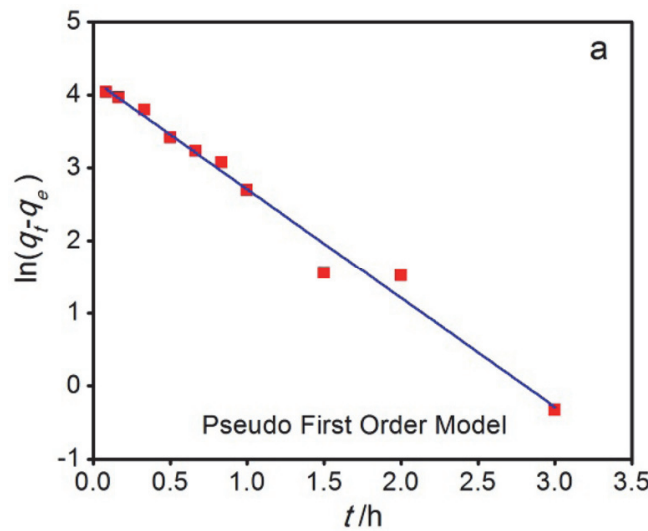

$$
t / q_{t}=1 /\left(k_{2} q_{\mathrm{e}}^{2}\right)+t / q_{\mathrm{e}}
$$

式中, $q_{t}$ 为 $t$ 时刻的吸附量, $\mathrm{mg} / \mathrm{g} ; q_{\mathrm{e}}$ 为平衡吸附量, $\mathrm{mg} / \mathrm{g}$; $k_{1}$ 为假一级反应速率常数, $\mathrm{h}^{-1} ; k_{2}$ 为假二级反应速率常 数, $\mathrm{g} /(\mathrm{mg} \cdot \mathrm{h}) ; t$ 为吸附时间.

方程拟合结果如图 4 和表 1 所示. 其中的速率常数 $k$ 和平衡吸附量 $q_{\mathrm{e}}$ 通过直线的斜率和截距得到. 由表 1 可知, 假一级动力学模型的相关系数 $\left(R^{2}=0.982\right)$ 及假二 级动力学模型的相关系数 $\left(R^{2}=0.998\right)$ 均大于 0.98 , 但假 二级动力学模型的平衡吸附量拟合结果 $\left(q_{\mathrm{e}}=109.8\right.$ $\mathrm{mg} / \mathrm{g}$ ) 非常接近时间动力学实验测得的平衡吸附容量 $\left(q_{\mathrm{e}}^{\exp }=110.8 \mathrm{mg} / \mathrm{g}\right)$, 而假一级动力学拟合结果 $\left(q_{\mathrm{e}}=\right.$ $67.36 \mathrm{mg} / \mathrm{g}$ )偏离了实验结果, 说明假二级动力学模型更 适合描述 CaAl-LDH 吸附磷酸根的动力学过程 ${ }^{[34]}$, 化学 吸附可能是 CaAl-LDH 吸附磷酸根的速率控制步骤 ${ }^{[12]}$.

\section{3 吸附等温线}

动力学实验得到的平衡吸附量不能真实反映吸附 剂的最大吸附容量. 为此进行了 CaAl-LDH 吸附磷酸根 的等温吸附实验(结果见图 5), 考察 CaAl-LDH 的最大吸 附容量及热力学行为. 由图 5 可知, 15 和 $25{ }^{\circ} \mathrm{C}$ 时, 不同 磷浓度下吸附量基本不变, $25{ }^{\circ} \mathrm{C}$ 时的最大吸附量略高 于 $15{ }^{\circ} \mathrm{C}$; 当温度升高至 $35{ }^{\circ} \mathrm{C}$ 时, 不同磷浓度下吸附量 均有增加, 最大吸附量比其它温度时最大吸附量提高 $12 \%$, 说明 CaAl-LDH 吸附磷酸盐可能是一个吸热过程.

运用 Langmuir 和 Freundlich 模型描述 CaAl-LDH 等 温 $\left(35{ }^{\circ} \mathrm{C}\right)$ 吸附磷酸根的过程. Langmuir 模型设定吸附过 程是吸附剂均质表面的单层吸附，且吸附质分子之间没 有相互作用; Freundlich 模型是一个经验方程，通常用于

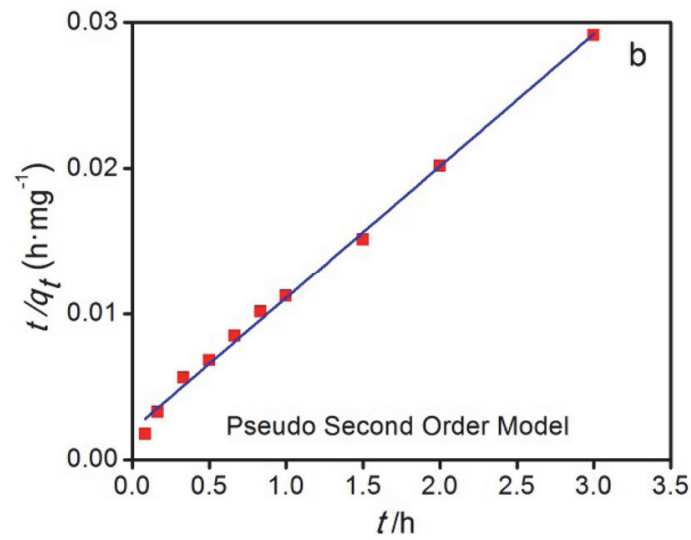

图 4 假一级 (a)、假二级 (b)反应动力学方程拟合 CaAl-LDH 吸附磷酸根动力学过程

Figure 4 Phosphate adsorption kinetic process on CaAl-LDH fitted by pseudo-first (a) and pseudo-second-order (b) model equations

表 1 吸附动力学模型和吸附等温线模型及其拟合常数

Table 1 Kinetic and isotherm models for phosphate adsorption on CaAl-LDH and its constants

\begin{tabular}{lll}
\hline 模型种类 & 模型常数 & 相关系数 $\left(R^{2}\right)$ \\
\hline 假一级动力学模型(Pseudo-first-order) & $q_{\mathrm{e}}=67.36 \mathrm{mg} / \mathrm{g}, k_{1}=0.651 \mathrm{~h}^{-1}$ & 0.982 \\
假二级动力学模型(Pseudo-second-order) & $q_{\mathrm{e}}=109.8 \mathrm{mg} / \mathrm{g}, k_{2}=0.041 \mathrm{~g} /(\mathrm{mg} * \mathrm{~h})$ & 0.998 \\
Langmuir 模型 & $q_{\max }=162.3 \mathrm{mg} / \mathrm{g}, K_{\mathrm{L}}=75.18 \mathrm{~L} / \mathrm{mg}$ & 0.997 \\
Freundlich 模型 & $n=4.03, K_{\mathrm{F}}=172.4 \mathrm{mg}^{(1-n)} \mathrm{L}^{n} / \mathrm{g}$ & 0.788 \\
\hline
\end{tabular}




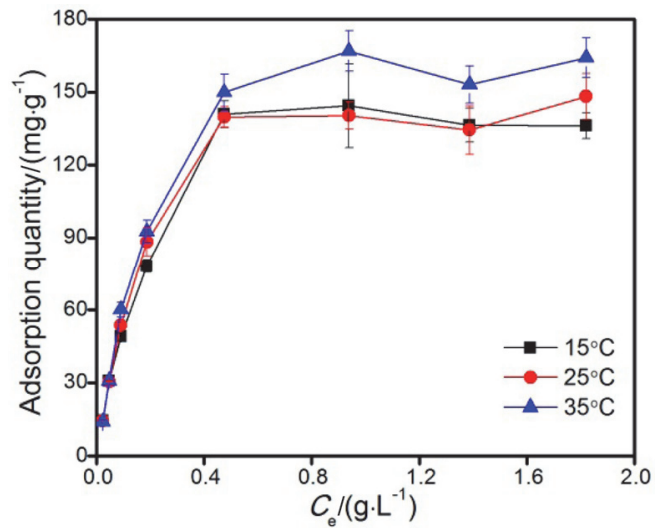

图 5 不同温度梯度下 CaAl-LDH 等温吸附磷酸盐结果

Figure 5 Phosphate adsorption isotherms by CaAl-LDH under different temperature gradients

描述异质表面的多层化学吸附 ${ }^{[22,35]}$. 分别用 Langmuir $(3)^{[36]}$ 和 Freundlich $(4)^{[22]}$ 方程拟合等温吸附实验数据, 方程的线性形式如下所示:

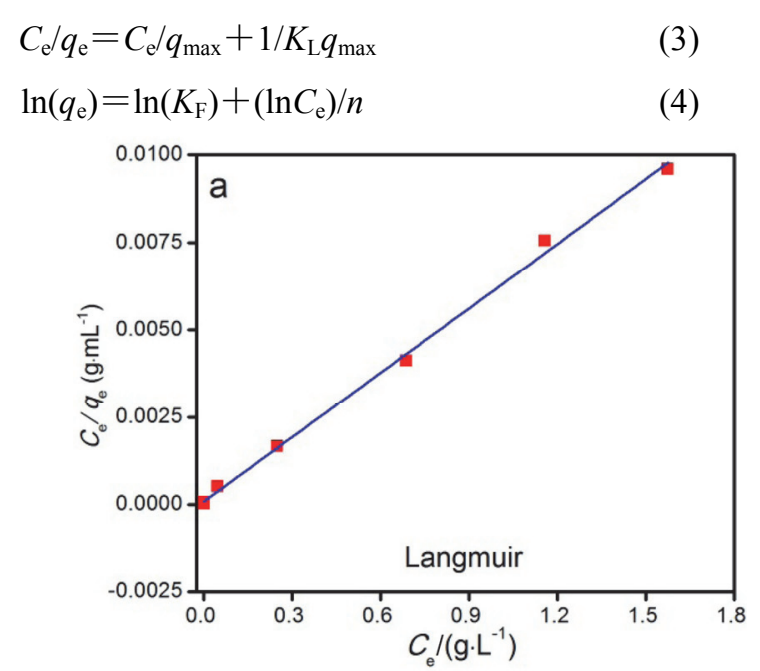

式中, $C_{\mathrm{e}}$ 为平衡溶液磷浓度, $\mathrm{g} / \mathrm{L} ; q_{\mathrm{e}}$ 为平衡吸附量, $q_{\text {max }}$ 为最大吸附量, $\mathrm{mg} / \mathrm{g} ; K_{\mathrm{L}} 、 K_{\mathrm{F}}$ 分别为两模型方程中与吸附 能有关的常数, $n$ 为常数.

图 6(a)、(b)分别为 Langmuir 和 Freundlich 模型的拟 合结果，拟合常数(见表 1)通过斜率和截距得到. 根据表 1 中拟合相关系数 $\left(R^{2}\right)$ 可知, Langmuir 模型 $\left(R^{2}=0.998\right)$ 拟 合效果优于 Freundlich 模型 $\left(R^{2}=0.788\right), \mathrm{CaAl}-\mathrm{LDH}$ 等温 吸附磷酸根的过程可用 Langmuir 模型描述. 可见, 吸附 过程可能是吸附剂均质表面的单层化学吸附. 本材料具 体的吸磷机理有待进一步研究. Langmuir 方程计算得出 的最大吸附容量 $\left(q_{\max }=162.3 \mathrm{mg} / \mathrm{g}\right)$ 与实际实验数据吻合 $\left(q_{\text {max }}^{\exp }=166.9 \mathrm{mg} / \mathrm{g}\right)$. 目前已知的其他类型吸附剂的吸 磷容量较低(见表 2), 非类水滑石材料的吸附容量一般 仅为 $0.56 \sim 28.1 \mathrm{mg} / \mathrm{g}^{[37,38]}, 300{ }^{\circ} \mathrm{C}$ 高温煅烧的 $\mathrm{ZnAl}-$ LDH 吸附容量只有 $72.46 \mathrm{mg} / \mathrm{g}^{[25]}$, 本实验材料 CaAl$\mathrm{LDH}$ ，在 $60{ }^{\circ} \mathrm{C}$ 下干燥制得，由 Langmuir 模型算得的吸 附容量高达 $162.3 \mathrm{mg} / \mathrm{g}$. 因此, 本材料具有高效的吸磷 能力.

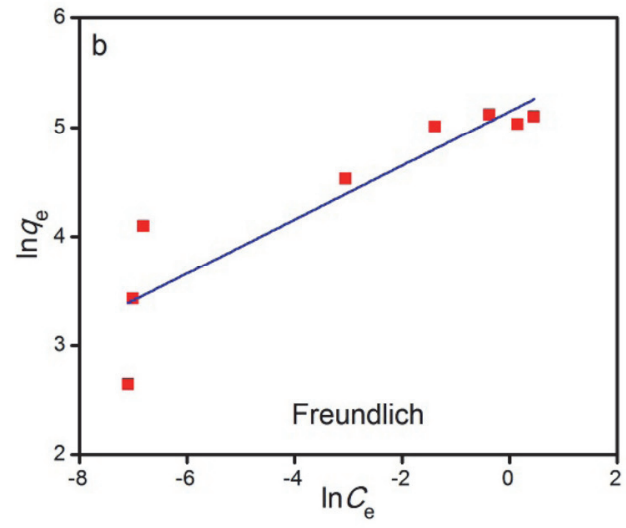

图 6 Langmuir 模型(a)和 Freundlich 模型(b)拟合 CaAl-LDH 等温吸附磷酸根结果

Figure 6 Adsorption isotherm fitted by Langmuir (a) and Freundlich (b) model

表 2 不同吸附剂的吸磷容量比较

Table 2 Phosphate adsorption capacities of different adsorbents

\begin{tabular}{|c|c|c|c|}
\hline 吸附剂种类 & 吸附剂处理及吸附液 pH & 吸磷容量 $q_{\mathrm{e}} /\left(\mathrm{mg} \cdot \mathrm{g}^{-1}\right)$ & 数据来源 \\
\hline ZnAl-LDH & $60{ }^{\circ} \mathrm{C}$ 干燥 & 30.29 & {$[10]$} \\
\hline MgAlFe-LDH & $300{ }^{\circ} \mathrm{C}$ 煅烧 & 27.03 & {$[13]$} \\
\hline MgAl-LDH & $500{ }^{\circ} \mathrm{C}$ 煅烧, $\mathrm{pH}=5 \sim 7$ & 47.1 & [39] \\
\hline ZnAlLa-LDH & $80{ }^{\circ} \mathrm{C}$ 干燥、 $300{ }^{\circ} \mathrm{C}$ 㷽烧 & $35.15,52.02$ & {$[14]$} \\
\hline ZnAl-LDH & $300{ }^{\circ} \mathrm{C}$ 炦烧, $\mathrm{pH}=7$ & 72.46 & {$[25]$} \\
\hline ZnAl-LDH & $300{ }^{\circ} \mathrm{C}$ 煅烧, $\mathrm{pH}=6 \sim 9$ & 40.77 & {$[40]$} \\
\hline ZnAlFe-LDH & $80{ }^{\circ} \mathrm{C}$ 干燥 & 51.81 & {$[41]$} \\
\hline 红壤 & $\mathrm{pH}=7$ & 0.56 & {$[37]$} \\
\hline 氧化镧改性膨胀石墨 & & 10.3 & {$[42]$} \\
\hline 氧化锆纳米颗粒 & & 28.1 & {$[38]$} \\
\hline $\mathrm{Fe}_{3} \mathrm{O}_{4}$ 纳米颗粒 & $\mathrm{pH}=2.77$ & 3.65 & {$[43]$} \\
\hline 黄铁矿 & $600{ }^{\circ} \mathrm{C}$ 煅烧 & 5.36 & {$[44]$} \\
\hline CaAl-LDH & $60{ }^{\circ} \mathrm{C}$ 干燥, $\mathrm{pH}=4 \sim 7$ & 162.3 & 本研究 \\
\hline
\end{tabular}




\section{4 竞争离子对 CaAl-LDH 吸附磷酸根的影响}

实际污水废水是含有多种竞争阴离子的体系, 吸附 剂会根据亲和力及离子浓度等因素选择性的与吸附质 结合 ${ }^{[27,45]}$. 通过磷酸根与几种竞争阴离子的不同组合, 考察竞争阴离子对 $\mathrm{CaAl}-\mathrm{LDH}$ 吸附磷酸根的干扰作用. 结果如表 3 所示, $\mathrm{CO}_{3}^{2-}$ 与磷酸根共存时, 对 CaAl-LDH 吸附磷酸根的干扰作用最大，此时的吸附率仅为 $48.7 \%$; $\mathrm{NO}_{3}^{-}$与磷酸根共存时, 对 $\mathrm{CaAl}-\mathrm{LDH}$ 吸附磷酸根的干 扰作用最小, 此时的吸附率达 $91.3 \%$, 与仅含有磷酸根 时的吸附率 $92.8 \%$ 相差很小. 竞争阴离子对 $\mathrm{CaAl}-\mathrm{LDH}$ 吸附磷酸根的干扰作用由强到弱为 $\mathrm{CO}_{3}^{2-}>\mathrm{SO}_{4}^{2-}>$ $\mathrm{NO}_{3}^{-}$.

一定量的吸附剂(如 CaAl-LDH)上的吸附位点数量 有限, 各种阴离子通过竞争与这些位点结合 ${ }^{[46]}$. 与磷酸 根离子共存于同一吸附体系时, 高化合价的阴离子比单 价阴离子具有更强的干扰作用, 并且干扰作用顺序为 $\mathrm{SO}_{4}^{2-}>\mathrm{CO}_{3}^{2-}>\mathrm{NO}_{3}^{-[47,48]}$. 本研究结果显示 $\mathrm{CO}_{3}^{2-}$ 对 CaAl-LDH 吸附磷酸根的干扰大于 $\mathrm{SO}_{4}^{2-}$, 与 Koilraj 等 ${ }^{[27]}$ 的结果一致, 由于 $\mathrm{CO}_{3}^{2-}$ 与类水滑石层板间的强亲 和力, 导致 $\mathrm{CO}_{3}^{2-}$ 的干扰作用强于 $\mathrm{SO}_{4}^{2-}$. 另一方面, 由 表 3 可知, $\mathrm{Na}_{2} \mathrm{CO}_{3}$ 的 $\mathrm{pH}$ 值为 10.8, 显强碱性, 体系中含 有大量 $\mathrm{OH}^{-}$, 会与磷酸根竞争吸附位点, 导致磷酸根吸 附率降低 ${ }^{[34]}$.

表 3 加入不同竞争离子对吸附磷酸根的影响以及不同阴离子溶液的 $\mathrm{pH}$ 值

Table 3 Effects of adding different competitive anions on the sorption of phosphate and $\mathrm{pH}$ of stock anion solutions

\begin{tabular}{lll}
\hline 竞争阴离子组合 & 磷酸根吸附率/\% & $\mathrm{pH}$ \\
\hline $\mathrm{NaH}_{2} \mathrm{PO}_{4}$ & 92.8 & 5.4 \\
$\mathrm{NaH}_{2} \mathrm{PO}_{4}+\mathrm{Na}_{2} \mathrm{CO}_{3}$ & 48.7 & 10.8 \\
$\mathrm{NaH}_{2} \mathrm{PO}_{4}+\mathrm{Na}_{2} \mathrm{SO}_{4}$ & 83.9 & 7.1 \\
$\mathrm{NaH}_{2} \mathrm{PO}_{4}+\mathrm{NaNO}_{3}$ & 91.3 & 6.9 \\
\hline
\end{tabular}

\section{5 吸附剂投加量和吸附时间对 CaAl-LDH 去除实际 污水磷酸根的影响}

实际应用中, 为控制污水废水处理成本, 会选用合 适的吸附剂投加量; 为提高处理速度和效率会选择适当 的处理时间. 研究采用真实含磷污水(取自南京某化工 厂, 全磷含量约 $2.0 \mathrm{mg} / \mathrm{L})$, 考察吸附剂投加量和吸附时 间对 CaAl-LDH 去除磷酸根效果的影响.

\subsection{1 吸附剂投加量的影响}

吸附剂投加量对去除磷酸根的影响结果如图 7 所 示. 随着投加量的增加, 磷酸根的去除率逐渐增大, 并 且趋于稳定, 当吸附剂用量为 $2 \mathrm{~g} / \mathrm{L}$ 时, 达到最大去除 率 $(97.2 \%)$. 随着吸附剂投加量的逐渐增加, 因为一定体 积污水中磷酸根总量有限, 更多的吸附剂活性位点未能 被磷酸根占据, 导致单位吸附剂的磷酸根吸附容量降 低, 即吸附剂含磷量逐渐降低, 吸附剂的利用率降低.
根据工业废水排放标准中全磷含量不得高于 $0.5 \mathrm{mg} / \mathrm{L}$ 的规定 ${ }^{[49]}$, 当 CaAl-LDH 投加量为 $0.5 \mathrm{~g} / \mathrm{L}$ 时, 处理效果 即可达到排放标准. 可见，本材料吸附污水中磷酸根的 能力很强, 应用前景广阔.

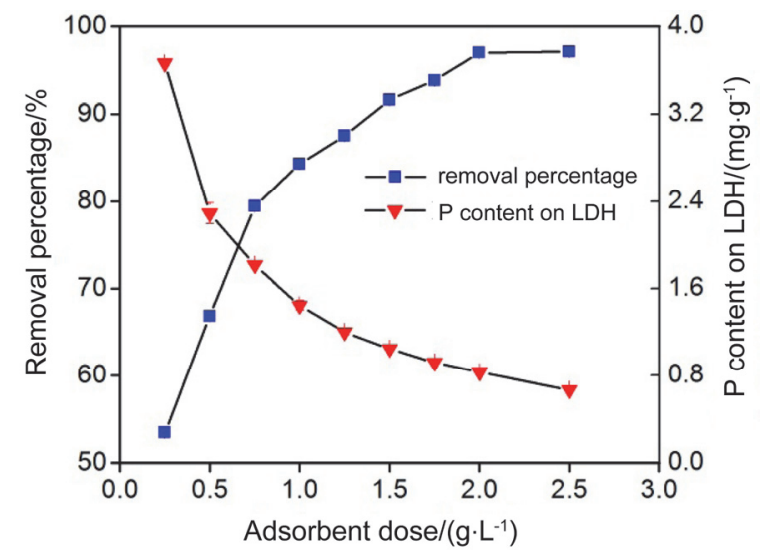

图 7 吸附剂投加量对 CaAl-LDH 吸附去除磷酸根的影响

Figure 7 Effect of adsorbent dosage on phosphate removal by CaAl-LDH

\subsection{2 吸附时间的影响}

图 8 所示为吸附时间对去除效果的影响，吸附剂用 量为 $2 \mathrm{~g} / \mathrm{L}$. CaAl-LDH 在 $5 \mathrm{~min}$ 中内吸附了污水中 $89.7 \%$ 的磷酸根, 此时溶液中的磷酸根浓度仅为 $0.18 \mathrm{mg} / \mathrm{L}$, 远 低于现行的污水排放标准中对磷酸根的限制 $(0.5$ $\mathrm{mg} / \mathrm{L})^{[49]}$. 随着吸附反应的进行, 溶液中的磷酸根浓度 逐渐下降, 但吸附速率也逐渐降低, 吸附过程在 $10 \mathrm{~h}$ 左 右达到平衡, 此时达到最大去除率 $98.5 \%$, 溶液中磷酸 根浓度仅为 $0.026 \mathrm{mg} / \mathrm{L}$, 低于造成水体富营养化的磷临 界浓度 $(0.03 \mathrm{mg} / \mathrm{L})^{[50]}$.

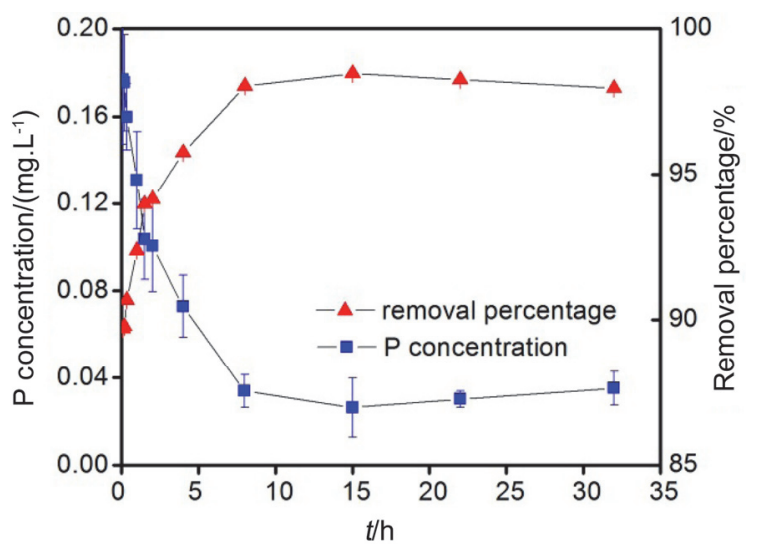

图 8 吸附时间对 CaAl-LDH 吸附磷酸根的影响

Figure 8 Effect of adsorption time on phosphate adsorption by CaAl-LDH

\section{3 结论}

(1)采用共沉淀法，简单快速地制备了高度热稳定 的 CaAl-LDH，对磷酸根具有极好的吸附效果，吸附容 量达 $162.3 \mathrm{mg} / \mathrm{g}$, 显著大于已见报道的各类吸附剂的吸 磷容量. 吸附过程分为快速和慢速两个阶段. 吸附动力 
学过程符合假二级动力学反应方程, 等温吸附规律符合 Langmuir 模型方程. CaAl-LDH 吸附磷酸盐是一个吸热 过程.

(2)溶液中含其他竞争阴离子时, 会干扰 CaAl-LDH 对磷酸根的吸附, 干扰作用由强到弱顺序为 $\mathrm{CO}_{3}^{2-}>$ $\mathrm{SO}_{4}^{2-}>\mathrm{NO}_{3}^{-}$. 增加吸附剂投加量会提高真实含磷污水 的磷酸根去除率, 但吸附剂含磷量降低, $10 \mathrm{~h}$ 左右达到 平衡. CaAl-LDH 对真实含磷污水的去除效果很好, 处理 后的污水可以达到排放标准.

\section{4 实验部分}

\section{1 分析仪器}

扫描电子显微 镜 (SEM, Hitachi S4800 Field-Emission)观察材料的形貌结构. 热重/差示扫描量 热分析(TG-DSC, NETZSCH STA409 PG/PC)测定材料 热稳定性. 电感耦合等离子体一原子发射光谱仪 (ICP-AES, IRIS Advantage, Thermo Jarrell Ash/Baird, Massachusetts, USA)测定材料组成含量( $\mathrm{P} 、 \mathrm{Ca} 、 \mathrm{Al}$ 等). METTLER TOLEDO SG78 型 $\mathrm{pH}$ 计测定溶液 $\mathrm{pH}$ 值(标 准缓冲液 $\mathrm{pH}$ 值分别为 4.01、7.00 和 9.21).

\subsection{CaAl-LDH 样品的制备}

共沉淀法制备 CaAl-LDH. 向含有 $0.2 \mathrm{~mol} \mathrm{NaOH}$ 溶 液的烧杯中, 同时缓慢加入 $0.25 \mathrm{~mol} \mathrm{CaCl}_{2}$ 溶液和 0.1 $\mathrm{mol} \mathrm{NaAlO}$ 溶液, $150 \mathrm{r} / \mathrm{min}$ 搅拌老化 $4 \mathrm{~h}$. 生成的胶状物 过滤、清洗数次后, 在 $60{ }^{\circ} \mathrm{C}$ 烘箱中干燥 $24 \mathrm{~h}$. 固体烘干 后研磨成粉, 得到 CaAl-LDH 样品.

\section{$4.3 \mathrm{CaAl}-\mathrm{LDH}$ 对磷酸根的批次吸附实验}

\subsection{1 时间动力学实验}

$0.100 \mathrm{~g} \mathrm{CaAl-LDH}$ 加入 $50 \mathrm{~mL}$ 离心管, 加入 40.0 $\mathrm{mL} 500 \mathrm{mg} / \mathrm{L}$ (以全 $\mathrm{P}$ 计) $\mathrm{NaH}_{2} \mathrm{PO}_{4}$ 溶液. $25{ }^{\circ} \mathrm{C}$ 恒温振荡, 按设定的时间间隔取样, $0.22 \mu \mathrm{m}$ 滤膜过滤后, 测定滤液 中磷浓度(ICP-AES) 并计算磷吸附量. 每个处理三次重 复(以下实验处理均三次重复).

\section{3 .2 等温吸附实验}

设置不同磷浓度溶液(用 $\mathrm{NaH}_{2} \mathrm{PO}_{4}$ 配制)进行等温 (15、25 和 $35{ }^{\circ} \mathrm{C}$ ) 吸附实验. CaAl-LDH (用量为 $1.5 \mathrm{~g} / \mathrm{L}$ ) 加入 $40 \mathrm{~mL}$ 含有不同浓度磷溶液的离心管. 相应温度下 振荡 $24 \mathrm{~h}$ 后，过滤测定磷浓度并计算磷吸附量.

\subsection{3 竞争离子对 CaAl-LDH 吸附磷酸根的影响实验}

实际含磷污水是一个多种阴离子共存的体系. 为研 究吸附体系中含有其它阴离子时, CaAl-LDH 吸附磷酸 根的效果, 分别设置含有 $100 \mathrm{mg} / \mathrm{L} \quad \mathrm{CO}_{3}^{2-}, \mathrm{SO}_{4}^{2-}$ 和 $\mathrm{NO}_{3}^{-}$三种阴离子的 $\mathrm{NaH}_{2} \mathrm{PO}_{4}(100 \mathrm{mg} / \mathrm{L})$ 溶液, 加入 $\mathrm{CaAl}-\mathrm{LDH}$ (用量为 $1.25 \mathrm{~g} / \mathrm{L}$ ), $25{ }^{\circ} \mathrm{C}$ 恒温振荡 $24 \mathrm{~h}$ 后, 过 滤测定磷浓度并计算磷吸附量.
4.3.4 吸附剂用量和吸附时间对 CaAl-LDH 吸附实际污 水磷酸根的影响实验

为研究吸附剂 CaAl-LDH 用量和吸附时间对实际废 水中磷酸根的吸附效果，分别设置不同吸附剂投加量 $(0.25 \sim 2.5 \mathrm{~g} / \mathrm{L})$ 及不同吸附时间 $(5 \mathrm{~min} \sim 32 \mathrm{~h})$ 的批次实 验. 吸附剂投加量实验时吸附时间为 $24 \mathrm{~h}$; 吸附时间实 验时吸附剂用量为 $2.0 \mathrm{~g} / \mathrm{L} .25{ }^{\circ} \mathrm{C}$ 恒温振荡相应时间，过 滤测定磷浓度并计算磷吸附量.

\section{References}

[1] Hart, M. R.; Quin, B. F.; Nguyen, M. L. J. Environ. Qual. 2004, 33, 1954.

[2] Drenkova-Tuhtan, A.; Mandel, K.; Paulus, A.; Meyer, C.; Hutter, F.; Gellermann, C.; Sextl, G.; Franzreb, M.; Steinmetz, H. Water Res. 2013, 47, 5670 .

[3] Gilbert, N. Nature 2009, 461, 1041.

[4] Anirudhan, T. S.; Noeline, B. F.; Mancihar, D. M. Environ. Sci. Technol. 2006, 40, 2740.

[5] Zhou, Q.; Wang, X. Z.; Liu, J. Y.; Zhang, L. Chem. Eng. J. 2012 200,619 .

[6] Ashekuzzaman, S. M.; Jiang, J. Q. Chem. Eng. J. 2014, 246, 97.

[7] Jiang, J. Q.; Wu, L. Desalin. Water Treat. 2012, 23, 49.

[8] Aguilar, M. I.; Saez, J.; Llorens, M.; Soler, A.; Ortuno, J. F. Water Res. 2002, 36, 2910.

[9] Tang, C. C.; Chen, H. M.; Liu, M.; Ye, X. Chem. Ind. Eng. Prog. 2015, 34, 245. (唐朝春, 陈惠民, 刘名, 叶金金, 化工进展, 2015, 34, 245.)

[10] Chen, X. L. M.S. Thesis, Beijing Forestry University, Beijing, 2014 (陈雪琳, 硕士论文, 北京林业大学, 北京, 2014.)

[11] Chitrakar, R.; Tezuka, S.; Sonoda, A.; Ooi, K.; Hirotsu, T. J. Colloid Interface Sci. 2005, 290, 45.

[12] Long, F.; Gong, J. L.; Zeng, G. M.; Chen, L.; Wang, X. Y.; Deng, J. H.; Niu, Q. Y.; Zhang, H. Y.; Zhang, X. R. Chem. Eng. J. 2011, 171, 448.

[13] Yin, L.; Lei, G. Y.; Li, C. J.; Liu, Z. J. Environ. Chem. 2012, 31, 1049. (印露, 雷国元, 李陈君, 刘志军, 环境化学, 2012, 31, 1049.)

[14] Cheng, X.; Huang, X. R.; Wang, X. Z.; Sun, D. Z. CIESC J. 2010, 61, 955. (程翔, 黄新瑞, 王兴祖, 孙德智, 化工学报, 2010, 61, 955.)

[15] Das, J.; Patra, B. S.; Baliarsingh, N.; Parida, K. M. Appl. Clay Sci. 2006, 32, 252.

[16] Sun, Z. W.; Xu, X. Q.; Hu, K.; Wang, B.; Xie, Y.; Huang, Z. Z. J. Shanghai Univ. of Eng. Sci. 2013, 27, 239. (孙照伟, 徐小强, 胡科, 王冰，谢园，黄中子，上海工程技术大学学报, 2013, 27, 239.)

[17] Baliarsingh, N.; Parida, K. M.; Pradhan, G. C. RSC Adv. 2013, 46, 23865.

[18] Zhou, J.; Xu, Z. P.; Qiao, S.; Liu, Q.; Xu, Y.; Qian, G. J. Hazard. Mater. 2011, 189, 586.

[19] Tsuji, H.; Fujii, S. Appl. Clay Sci. 2014, 99, 261.

[20] Cheng, X.; Huang, X. R.; Wang, X. Z.; Zhao, B. Q.; Chen, A. Y.; Sun, D. Z. J. Hazard. Mater. 2009, 169, 958.

[21] Theiss, F. L.; Ayoko, G. A.; Frost, R. L. J. Colloid Interface Sci 2013, 402, 114

[22] Cai, P.; Zheng, H.; Wang, C.; Ma, H.; Hu, J.; Pu, Y.; Liang, P. J. Hazard. Mater. 2012, 213, 100.

[23] Xing, K.; Wang, H. Z.; Guo, L. G.; Song, W. D.; Zhao, Z. P. Colloids Surf., A 2008, 328, 15.

[24] Yang, K.; Yan, Y. M.; Yu, S. J.; Shan, R. R.; Yu, H. Q.; Zhu, B. C.; Du, B. Sep. Purif. Technol. 2014, 124, 36.

[25] Chen, A. Y.; Cheng, X.; Huang, X. R.; Sun, D. Z. CIESC J. 2008, 59, 2270. (陈爱燕, 程翔，黄新瑞，孙德智，化工学报，2008，59, 2270.)

[26] Goh, K. H.; Lim, T. T.; Dong, Z. Water Res. 2008, 42, 1343.

[27] Koilraj, P.; Antonyraj, C. A.; Gupta, C.; Reddy, C. R. K.; Kannan, S. Appl. Clay Sci. 2013, 86, 111.

[28] Zhong, Q.; Li, H. Environ. Sci. 2014, 35, 1566. (钟琼, 李欢, 环境 科学, 2014, 35, 1566.)

[29] Li, L.; Zhang, C. Y.; Jiao, Q. Z.; Duan, X. Chin. J. Inorg. Chem. 
2001，31，113. (李蕾, 张春英, 矫庆泽, 段雪, 无机化学学报, 2001, 31, 113.)

[30] Zheng, J. H.; Tian, X. K.; Yu, K. C.; Wang, L. Y.; Yang, C.; He, M. Z. Acta Chim. Sinica 2006, 64, 2231. (郑建华, 田熙科, 俞开潮, 王 龙艳，杨超，何明中，化学学报, 2006, 64, 2231.)

[31] Zhang, X. Q.; Zeng, M. G.; Li, S. P. Acta Chim. Sinica 2013, 71, 246. (张晓晴, 曾美桂, 李淑萍, 化学学报, 2013, 71, 246.)

[32] Ho, Y. S.; McKay, G. Process Saf. Environ. Prot. 1998, 76, 332.

[33] Azizian, S. J. Colloid Interface Sci. 2004, 276, 47.

[34] Xing, K.; Wang, H. Z.; Guo, L. G.; Song, W. D.; Zhao, Z. P. Colloids Surf., A 2008, 328, 15.

[35] Novillo, C.; Guaya, D.; Avendano, A. A. P.; Armijos, C.; Cortina, J. L.; Cota,I. Fuel 2014, 138, 72.

[36] Cheng, X.; Huang, X. R.; Wang, X. Z.; Sun, D. Z. J. Hazard. Mater. 2010, $177,516$.

[37] Rout, P. R.; Bhunia, P.; Dash, R. R. Desalin. Water Treat. 2015, 54, 358.

[38] Chen, L.; Zhao, X.; Pan, B. C.; Zhang, W. X.; Hua, M.; Lv, L.; Zhang, W. M. J. Hazard. Mater. 2015, 284, 35.

[39] Zhao, W.; Chen, Y. N. Appl. Chem. Ind. 2013, 42, 450. (赵维, 陈佑 宁, 应用化工, 2013, 42, 450.)
[40] Sun, D. Z.; Huang, X. R.; Cheng, X.; Chen, A. Y. Journal of Beijing Forestry University 2009, 31, 128. (孙德智, 黄新瑞, 程翔, 陈爱 燕, 北京林业大学学报, 2009, 31, 128.)

[41] Yin, L.; Lei, G. Y.; Li, C. J.; Liu, Z. J. Industrial Safety and Environmetal Protection 2013, 39, 1. (印露, 雷国元, 李陈君, 刘志军, 工业安全与环保, 2013, 39, 1.)

[42] Zhang, L.; Gao, Y.; Li, M. X.; Liu, J. Y. Environ. Technol. 2015, 36 , 1016.

[43] Tu, Y. J.; You, C. F.; Chang, C. K.; Chen, M. H. J. Taiwan Inst. Chem. Eng. 2015, 46, 148.

[44] Chen, T. H.; Wang, J. Z.; Wang, J.; Xie, J. J.; Zhu, C. Z.; Zhan, X. M. Int. J. Environ. Sci. Technol. 2015, 12, 885.

[45] Islam, M.; Patel, R. J. Hazard. Mater. 2009, 169, 524.

[46] Kushwaha, S.; Soni, H.; Ageetha, V.; Padmaja, P. Crit. Rev. Environ. Sci. Technol. 2013, 43, 443

[47] Yang, L.; Shahrivari, Z.; Liu, P. K. T.; Sahimi, M.; Tsotsis, T. T. Ind. Eng. Chem. Res. 2005, 44, 6804.

[48] You, Y. W.; Vance, G. F.; Zhao, H. T. Appl. Clay Sci. 2001, 20, 13.

[49] Li, W. W.; Sheng, G. P.; Zeng, R.; Liu, X. W.; Yu, H. Q. Environ. Sci. Pollut. Res. 2012, 19, 1422

[50] Xiong, J. B.; Mahmood, Q. Desalination 2010, 259, 59.

(Cheng, F.) 